\title{
Gold/Tin Oxide Nanocomposite by Nanojoining
}

\author{
Geetika Bajaj and R.K. Soni* \\ Department of Physics, Indian Institute of Technology Delhi, New Delhi-110016, India
}

\begin{abstract}
In this paper we report synthesis of $\mathrm{Au}-\mathrm{SnO}_{2}$ nanocomposite by nano-joining. It involves synthesis of $\mathrm{Au}$ and $\mathrm{SnO}_{2}$ colloids separately followed by post irradiation of mixed Au-SnO${ }_{2}$ colloids. We have used $532 \mathrm{~nm}$ and $1064 \mathrm{~nm}$ wavelengths of a pulsed Nd:YAG laser and different post irradiation time. The post irradiation by $532 \mathrm{~nm}$ laser light results in formation of $\mathrm{Au}-\mathrm{SnO}_{2}$ nanocomposite by nanojoining. Distinct gold nanoparticles along with the $\mathrm{SnO} \mathrm{O}_{2}$ aggregates are observed in case of post irradiation by $1064 \mathrm{~nm}$ showing no nanojoining effect. Our results indicate that nanojoining is a wavelength selective process. The X-ray Photoelectron Spectroscopy (XPS) indicates negative binding energy shift of $\mathrm{Au} 4 \mathrm{f}$ peak whereas the position of $\mathrm{Sn} 3 \mathrm{~d}$ peak remains unaffected after the post irradiation process. The invariance of Sn $3 \mathrm{~d}$ peak after post irradiation indicates no change in the valence state of Sn. A red shift with respect to the plasmon resonance peak of gold colloids is observed in $\mathrm{Au}-\mathrm{SnO}_{2}$ nanocomposite.
\end{abstract}

PACS: 52.38.Mf; 61.46.-w; 81.07. -b.

Keywords: Nanocomposites, nanojoining, laser ablation, plasmon resonance.

\section{INTRODUCTION}

The nanocomposite materials synthesis has gained considerable attention from material scientists because various physical properties can be tailored in these nanocomposites systems for specific applications. The field of nanocomposite materials has been widely recognized as one of the most promising and rapidly emerging research areas. The constituents of a nanocomposite have different structures, compositions and properties. Thus, the materials built from them can be multifunctional. The single component building blocks with dimensions in the nanometer size make it possible to design and create new materials with unprecedented flexibility. Recent reports have shown that these nanocomposites materials offer enhanced properties and improved characteristics as compared to their single components counterpart materials [1-4]. Apart from the properties of individual components in a nanocomposite, interfaces can also play an important role in enhancing or limiting the overall properties of the system.

The immense potential of $\mathrm{Au}-\mathrm{SnO}_{2}$ nanocomposite in a broad range of applications has recently created a lot of interest in this material system. They can be used as nanocapacitors with high electronic capacitance and low leakage rates [5]. They are used for efficient catalytic activity [6] and for sensor applications [7]. Recently, pulsed laser ablation in liquid (PLAL) has been widely applied for the generation of nanoparticles (NPs). PLAL has proved to be an efficient and versatile physical method for fabrication of nanomaterials [8]. It offers many advantages over the conventional wet chemical methods for synthesis of colloidal NPs. In comparison to wet chemical techniques, in which the

*Address correspondence to this author at the Department of Physics, Indian Institute of Technology Delhi, New Delhi-110016, India;

Tel: +91 1126591304; Fax: +91 1126581114;

E-mail: ravisoni@physics.iitd.ac.in synthesized NPs are significantly surface contaminated and require secondary treatments, PLAL is a chemically clean synthesis process. It is capable of generating NPs in the form of colloidal without the formation of chemical by-products. This technique possesses flexibility in controlling the properties of NPs by appropriate choice of laser parameters $[9,10]$ and surrounding liquid $[10,11]$.

In this paper, we have studied the structural, compositional and optical properties of $\mathrm{Au}-\mathrm{SnO}_{2}$ nanocomposites obtained by two-step method. At first, laser ablation of pure single metal targets is performed in deionized water to form individual gold and tin oxide colloidal. The two colloidal solutions are mixed and post irradiated by pulsed laser beam to form $\mathrm{Au}-\mathrm{SnO}_{2}$ nanocomposite by nanojoining effect. The nanojoining involves selective heating and melting of one of the components by using $532 \mathrm{~nm}$ laser wavelength in a mixed colloidal solution whereas the other component remains unaffected in the post irradiation [12]. The heated or melted gold particles can join at the interface with $\mathrm{SnO}_{2} \mathrm{NPs}$ which are relatively at lower temperature.

\section{EXPERIMENTAL}

A gold target $\left(99.9 \%\right.$ pure) immersed in $10 \mathrm{~cm}^{3}$ of deionized water was kept in a glass vial. The gold target was irradiated with the second harmonic of a pulsed Nd:YAG laser $(532 \mathrm{~nm})$ with pulse repetition rate $10 \mathrm{~Hz}$ and pulse width $5 \mathrm{~ns}$. The laser beam was focused on the target surface using a lens of focal length $150 \mathrm{~mm}$. The ablation was carried out for 15 minutes with laser energy $36 \mathrm{~mJ} /$ pulse. A tin target (99.9\%) immersed in $10 \mathrm{~cm}^{3}$ of deionized water was ablated separately for 30 minutes by the same laser at a lower energy $18 \mathrm{~mJ} /$ pulse. The lower laser energy was used for ablation of tin owing to lower melting point of tin as compared to gold. The synthesized $\mathrm{Au}$ and $\mathrm{SnO}_{2}$ colloidal solution were then mixed and post irradiated by fundamental $(1064 \mathrm{~nm})$ or second harmonic $(532 \mathrm{~nm})$ wavelength light of 
Nd:YAG laser at the energy of $14 \mathrm{~mJ} /$ pulse for $10 \mathrm{~min}$. The suspensions were dropped on copper mesh covered with an amorphous carbon film and dried at room temperature for transmission electron microscopy (TEM) measurements. For compositional analysis of the synthesized nanocomposite, the sample was centrifuged and the collected powder was dried on a quartz substrate for X-ray Photoelectron Spectroscopy measurements using SPECS XPS system attached with PHOIBOS. The survey scan was obtained at a step size $1 \mathrm{eV}$. The samples were sputtered using $\mathrm{Ar}$ ion beam of energy $5 \mathrm{keV}$ for $2 \mathrm{~min}$. The HRXPS of photoelectron peaks of main elements was then obtained at a step size of $0.05 \mathrm{eV}$. The relative peak shifts due to the electrostatic charge-up were corrected by fixing the peak position of the carbon (C1s peak) at $284.6 \mathrm{eV}$. The optical absorption of the samples was recorded using Perkin Elmer Lambda 35 spectrometer with resolution of $1 \mathrm{~nm}$.

\section{RESULTS AND DISCUSSION}

The gold NPs are prepared by pulsed laser ablation of gold target in deionized water. The solution turned red after the ablation of gold target. The formation of gold colloidal is confirmed by appearance of characteristic plasmon peak at $520 \mathrm{~nm}$ in the absorption spectrum (discussed later). The tin oxide NPs are prepared by pulsed laser ablation of tin target in deionized water. The solution remained colorless but appeared turbid after the ablation process. The formation of tin oxide NPs can be explained on the basis of pulsed laser induced reactive quenching in liquid medium [13]. In case of laser ablation of transition metal targets the surrounding liquid not only confines the ablated species at the liquidsolid interface but also acts as a mediator for chemical reaction at the liquid-solid interface. Strong chemical reactions can occur between the highly energetic species ablated from the target and the surrounding liquid molecules. The tin oxide NPs can be formed by the chemical reaction between active tin species and water molecules.

After the formation of $\mathrm{Au}$ and $\mathrm{SnO}_{2}$ colloids, we mixed the colloids in equal volumetric ratio and post irradiated with $1064 \mathrm{~nm}$ or $532 \mathrm{~nm}$ wavelength of Nd:YAG laser. The TEM micrographs of the samples are shown in Fig. (1). The $\mathrm{pH}$ value of the oxide NPs in pure water is below the isoelectric point indicating that the particles are positively charged at the surface [13]. We have carried out ablation in the absence of any surfactant so the positively charged NPs initially repel each other due to electrostatic repulsive forces. The repulsive forces promote further oxidation by attachment of the hydroxyl which causes decrease in surface charge. Due to reduced surface charge the tin oxide NPs can coalesce to form $\mathrm{SnO}_{2}$ aggregates. The TEM images of samples post irradiated by $1064 \mathrm{~nm}$ laser wavelength showed distinct gold NPs in between the $\mathrm{SnO}_{2}$ aggregates. In case of the samples post irradiated by $532 \mathrm{~nm}$ laser wavelength, change in particle morphology was observed in TEM micrographs. From the TEM micrographs, it is evident that the molten gold NPs have joined with $\mathrm{SnO}_{2} \mathrm{NPs}$ to form of $\mathrm{Au}-\mathrm{SnO}_{2}$ nanocomposite by nano-joining. The elongated rod like structure clearly shows the nano-joining effect. From the TEM micrographs it can be concluded that after post irradiation by $1064 \mathrm{~nm}$ the particles remain distinct and no nano-joining effect was observed. The post irradiation by
$532 \mathrm{~nm}$, on the other hand, results in formation of $\mathrm{Au}-\mathrm{SnO}_{2}$ nanocomposite by nano-joining and clearly shows the wavelength selective nature of the joining process. In case of post irradiation by $532 \mathrm{~nm}$ wavelength, strong laser light interaction with gold NPs can occur. The gold NPs in mixed solution absorb photons efficiently as the laser wavelength is near the plasmon absorption band. The melting point of gold NPs decreases with decrease in particle size and is sufficiently less in comparison of bulk gold [14]. During a single laser pulse, one gold NP is considered to absorb many photons successively and the temperature reaches as high as the melting point [15]. Thus absorption of photons can lead to heating and even melting of gold NPs. The selective heating of the gold NPs due to near resonance condition with surface plasmon absorption (SPA) band around $520 \mathrm{~nm}$ creates liquid suspension in the solution during the post irradiation process. The heating effect of the tin oxide NPs in solution is relatively weak due to extremely low absorption in the visible range. Tin oxide is wide bandgap (3.6 eV) semiconductor and its band edge absorption occurs at 345 $\mathrm{nm}$ which blue shifts in nanoparticles due to reduced size effect. The tin oxide nanoparticles show strong absorption in UV range and have extremely weak absorption in visible range. The tin oxide NPs remain in solid form during the laser irradiation. The liquid gold suspension in the colloidal solution joins the solid tin oxide NPs resulting in the formation of $\mathrm{Au}-\mathrm{SnO}_{2}$ nanocomposite [12]. For the samples prepared by post irradiation by $1064 \mathrm{~nm}$ laser wavelength, the joining effect was weak owing to weak absorption of both $\mathrm{Au}$ NPs and $\mathrm{SnO}_{2} \mathrm{NPs}$ at this wavelength.

The effect of post-irradiation time on the morphology of the $\mathrm{Au}-\mathrm{SnO}_{2}$ nanocomposites was investigated by TEM. The TEM micrographs of the $\mathrm{Au}-\mathrm{SnO}_{2}$ nanocomposites are shown in Fig. (2). The Au- $\mathrm{SnO}_{2}$ nanocomposites synthesized using $532 \mathrm{~nm}$ showed a drastic change in particle morphology from spherical to elongated nanoparticles with increasing post-irradiation time. For post-irradiation time of 5 min the particles are mostly spherical in shape with an average particle size of $4 \mathrm{~nm}$ and are interconnected. We observe a change in circularity with an increase in irradiation time. For the post irradiation time of 10 mins some spherical nanoparticles with an average size of about $7 \mathrm{~nm}$ were observed along with the elongated particles. The length of these elongated particles varies from $8 \mathrm{~nm}$ to $26 \mathrm{~nm}$. The nano-joining process is size selective in nature. The melting point of gold nanoparticles decreases appreciably with decrease in the particle size due to reduction in coordination number and cohesive energy of the surface atoms. During the post-irradiation process, the smaller nanoparticle can undergo complete melting whereas the larger particle undergo only partial melting or surface melting which indicates that the smaller particles have a stronger role in this process. Fig. (2) shows increased average particle size with increase in post-irradiation time due to laser induced thermal fusion of smaller particles. The change in morphology with increase post-irradiation time can be explained on the basis of fusion of photo-thermally melted gold nanoparticles. The particles can undergo either photo-thermal fusion or photofragmentation depending upon their state before irradiation. During the post-irradiation process the temperature of gold nanoparticles can reach much above the melting point and 

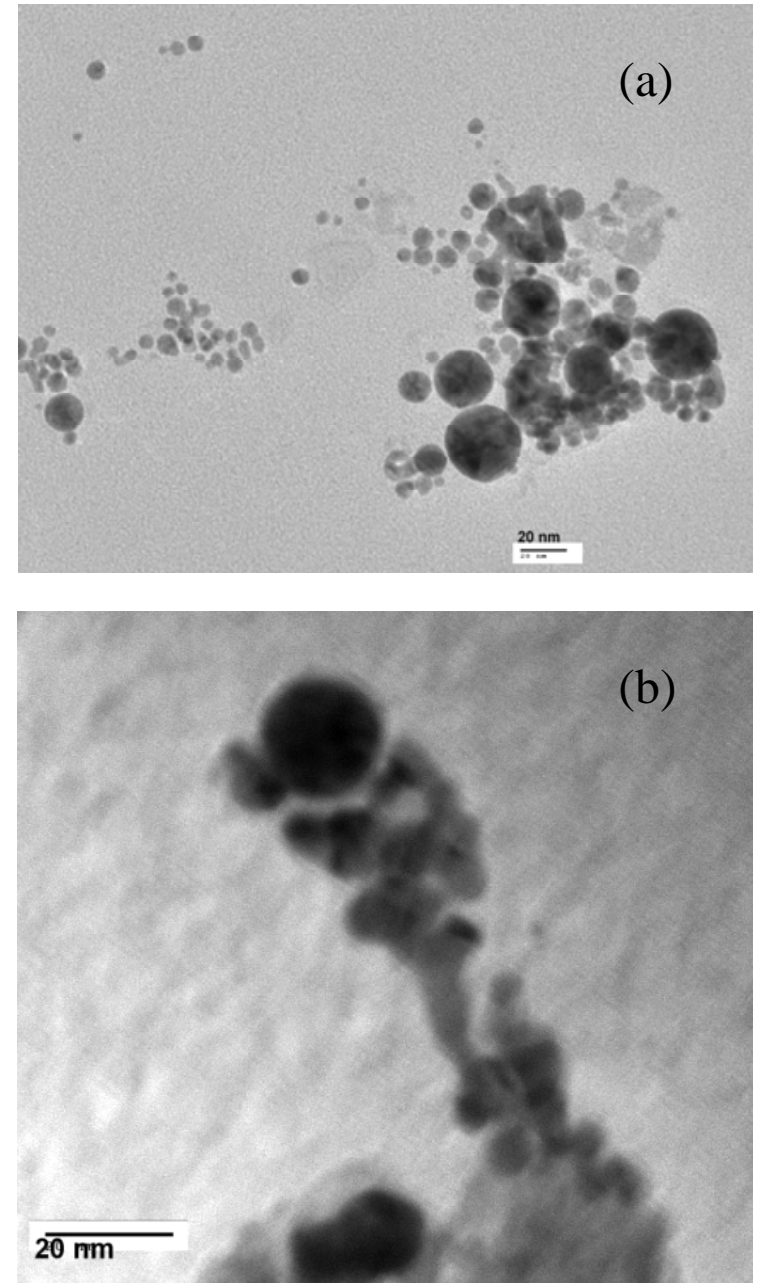

Fig. (1). High magnification TEM micrographs of the samples post irradiated at laser energy of the $14 \mathrm{~mJ} /$ pulse for $10 \mathrm{~min}$ by (a) 1064 nm (b) $532 \mathrm{~nm}$

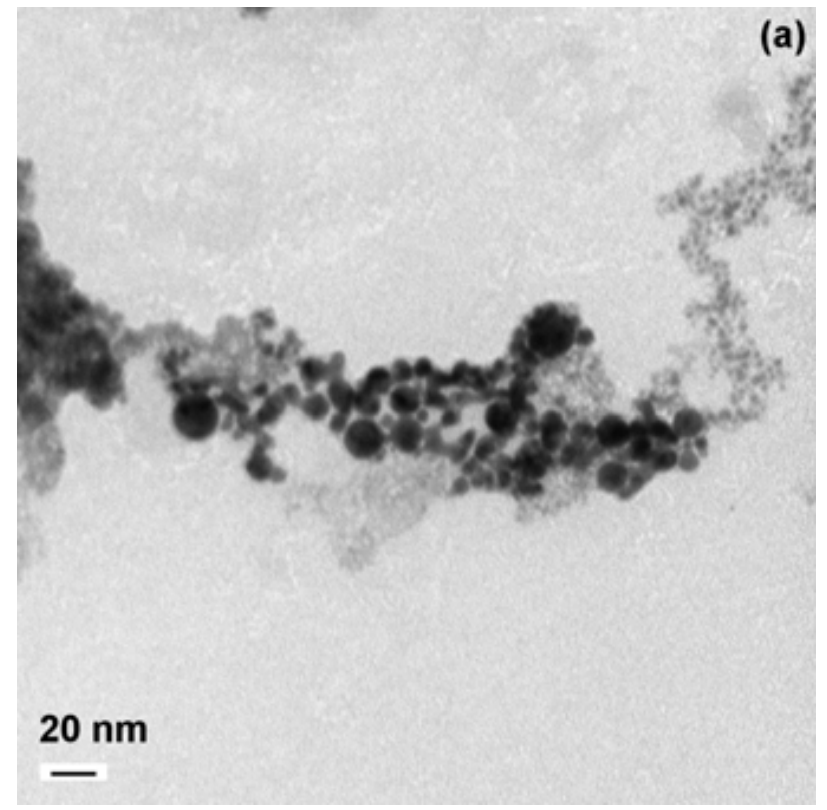

the heated particles can fuse together by encounter and coagulation. The initial agglomeration may enhance the intrinsic encounter rate of the nanoparticles. The laser induced fusion of these aggregates form larger particles on irradiation.

The XPS spectrum of the synthesized tin oxide NPs and $\mathrm{Au}-\mathrm{SnO}_{2}$ nanocomposite are shown in Fig. (3). The HRXPS of $\mathrm{Au} 4 \mathrm{f}$ and $\mathrm{Sn} 3 \mathrm{~d}$ peak is shown in insets. The spectrum of tin oxide shows the photoelectron peaks of $\mathrm{Sn}, \mathrm{O}, \mathrm{C}$, and weak Si peaks which came from the quartz substrate. The $\mathrm{Sn} 3 \mathrm{~d}_{5 / 2}$ peak was located at $486.6 \mathrm{eV}$ which corresponds to the binding energy of $\mathrm{Sn}$ in $\mathrm{SnO}_{2}$ [16]. The XPS spectrum of the $\mathrm{Au}-\mathrm{SnO}_{2}$ nanocomposite shows that the photoelectron peaks of $\mathrm{Au}, \mathrm{Sn}, \mathrm{O}, \mathrm{C}$, and weak Si peaks which came from the quartz substrate. The $\mathrm{Sn}_{3} \mathrm{~d}_{5 / 2}$ peak was located at 486.55 $\mathrm{eV}$ position. The invariance in Sn 3d peak positions shows that no change in the oxidation state of tin has occurred after the post irradiation process. It also confirms the fact that post irradiation process resulted in nano-joining and not in partial alloying of $\mathrm{Au}$ and $\mathrm{SnO}_{2} \mathrm{NPs}$.

The $4 \mathrm{f}_{7 / 2}$ peak in $\mathrm{Au}-\mathrm{SnO}_{2}$ nanocomposite was located at $83 \mathrm{eV}$. The $\mathrm{Au} 4 \mathrm{f}$ peak show a binding energy negative shift of $1 \mathrm{eV}$ with respect to bulk $\mathrm{Au}(84 \mathrm{eV})$. The binding energy of $\mathrm{Au} 4 \mathrm{f}$ peaks strongly depends on the chemical or electronic environment surrounding it. The shift of $\mathrm{Au} 4 \mathrm{f}$ binding energy can be ascribed to the small size of Au NPs $(<2 \mathrm{~nm})$ or to the electron structure difference between metal and the support [17]. In our case, the charge transfer from the $\mathrm{SnO}_{2}$ can be responsible for the observed negative shift. The difference in the work function of $\mathrm{Au}(5.42 \mathrm{eV})$ [18] and $\mathrm{SnO}_{2}(4.3 \mathrm{eV})$ [19] facilitates the electron transfer from $\mathrm{SnO}_{2}$ to $\mathrm{Au}[20]$ and the negative charging leads to the shift toward lower binding energies as compared to bulk gold.

The absorption spectra of the synthesized gold NPs, tin oxide NPs, $\mathrm{Au}-\mathrm{SnO}_{2}$ nanocomposite samples prepared by

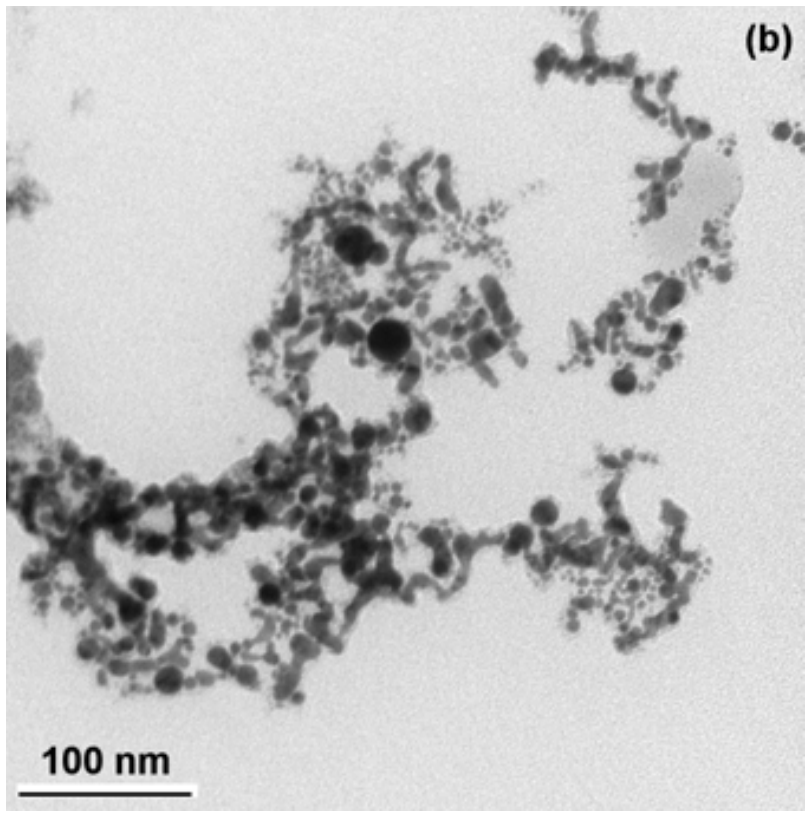

Fig. (2). TEM micrographs of the synthesized samples post-irradiation by $532 \mathrm{~nm}$ for time (a) $5 \mathrm{~min}$ and (b) $10 \mathrm{~min}$. 

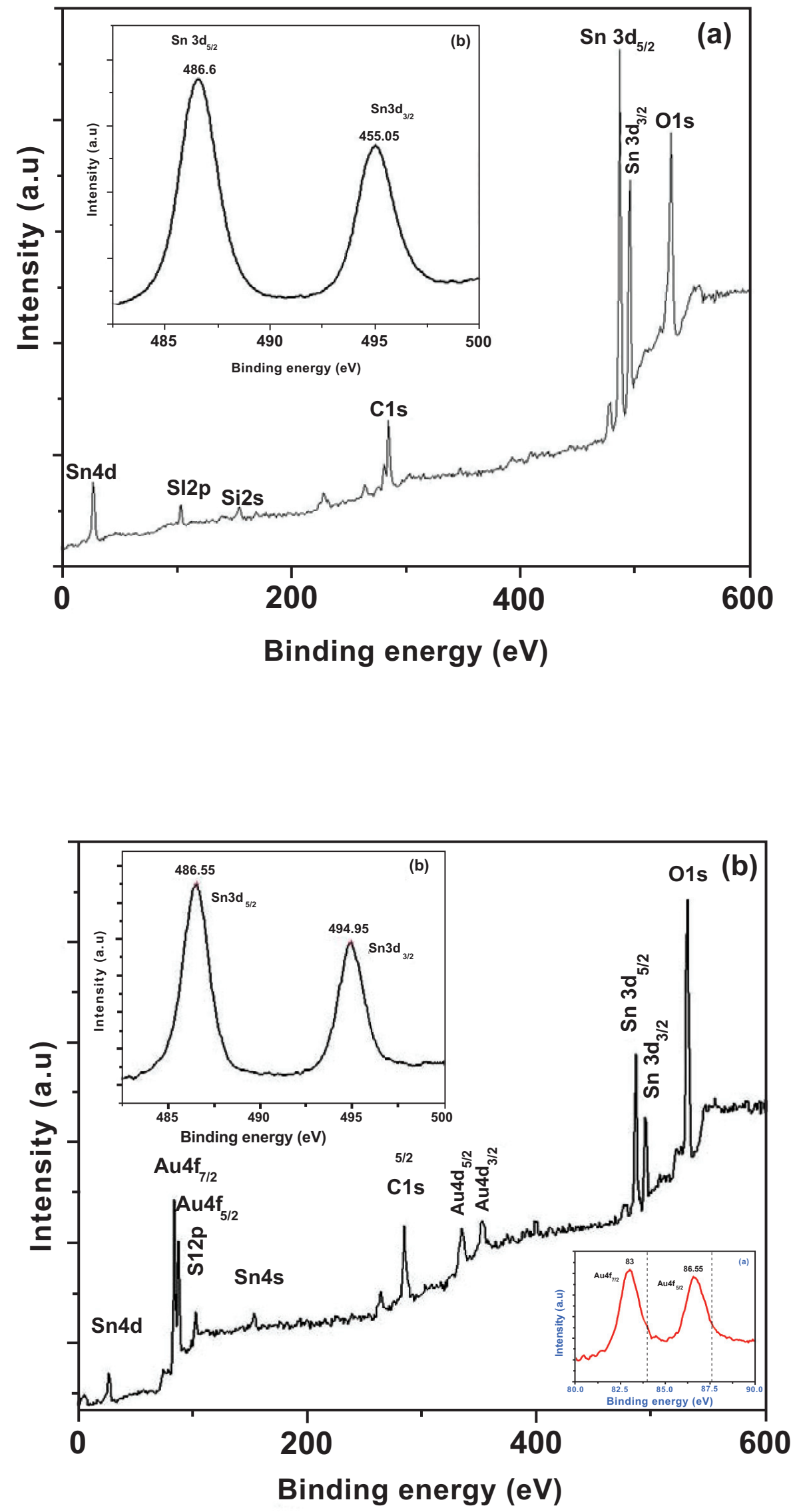

Fig. (3). XPS spectrum of the (a) tin-oxide nanoparticles (b) Au-SnO 2 nanocomposite. The insets show the HRXPS of (a) Au4f region, where the dashed lines show the binding energies of bulk gold and (b) Sn3d region. 
post irradiation with $532 \mathrm{~nm}$ and $1064 \mathrm{~nm}$ laser wavelength are shown in Fig. (4). The absorption spectra of gold colloidal show characteristic surface plasmon absorption peak at $520 \mathrm{~nm}$. The tin oxide colloidal exhibits strong UV absorption and negligible absorption in visible range. The absorption spectra of the sample post irradiated by $1064 \mathrm{~nm}$ does not show any appreciable change whereas $\mathrm{Au}-\mathrm{SnO}_{2}$ nanocomposite shows a slight red shift and significant change in the line shape of the plasmon absorption band. The presence of plasmon peak in the spectrum of $\mathrm{Au}-\mathrm{SnO}_{2}$ nanocomposite also confirms the formation of nanocomposite as formation of nano alloy is characterized by a blue shift or complete disappearance of plasmon peak. The absorption results suggest the formation of an intermediate phase upon the nano-joining [21]. This intermediate phase is characterized by a red shift in absorption peak and the position of plasmon peak depends on the size of the synthesized composite nanoparticles. The slight red shift observed in $\mathrm{Au}-\mathrm{SnO}_{2}$ nanocomposite can be ascribed to an increase in particle size upon nano-joining.

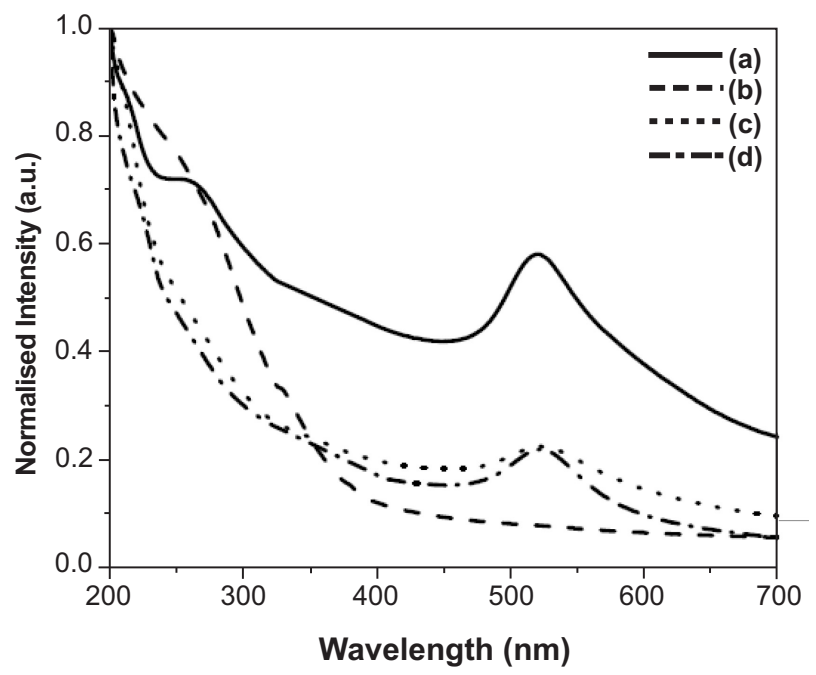

Fig. (4). Optical absorption spectra of (a) gold nanoparticles (b) tin oxide nanoparticles (c) $\mathrm{Au}-\mathrm{SnO}_{2}$ nanocomposite post irradiated by $532 \mathrm{~nm}$ (d) $\mathrm{Au}-\mathrm{SnO}_{2}$ nanocomposite post irradiated by $1064 \mathrm{~nm}$.

\section{CONCLUSION}

We have studied the effect of laser wavelength on the nano-joining process. Our results indicate that nano-joining is a wavelength dependent phenomenon. The formation of $\mathrm{Au}-\mathrm{SnO}_{2}$ nanocomposite by $532 \mathrm{~nm}$ laser post irradiation is due to near resonance condition with gold plasmon resonance band. By suitable choice of single component material and laser wavelength PLAL in combination with post irradiation can be used as a convenient synthesis route for the multicomponent materials by nano-joining.

\section{ACKNOWLEDGEMENTS}

This work was supported by DRDO, India research grant on Core-shell nanoparticles by pulsed laser ablation. One of the authors (G.B.) acknowledges the financial support provided by the University Grants Commission (UGC), India.

\section{REFERENCES}

[1] Zeng H, Sun S. Syntheses, properties, and potential applications of multicomponent magnetic nanoparticles. Adv Funct Mater 2008; 18: $391-400$

[2] Pan X, Fan Z, Chen W, Ding Y, Luo H, Bao X. Enhanced ethanol production inside carbon-nanotube reactors containing catalytic particles. Nat Mater 2007; 6: 507-11.

[3] Robel I, Bunker B, Kamat PV. Single-walled carbon nanotube-cds nanocomposites as light-harvesting assemblies: photoinduced charge-transfer interactions. Adv Mater 2005; 17: 2458-63.

[4] Komarnen S. Nanocomposites. J Mater Chem 1992; 2: 1219-30.

[5] Oldfield G, Ung T, Mulvaney P. Au@ $\mathrm{SnO}_{2}$ core-shell nanocapacitors. Adv Mater 2000; 12: 1519-22.

[6] Yu K, Wu Z, Zhao Q, Li B, Xie Y. High-temperature-stable $\mathrm{Au} @ \mathrm{SnO}_{2}$ core/shell supported catalyst for $\mathrm{CO}$ oxidation. J Phys Chem C 2008; 112: 2244-7.

[7] Bahrami B, Khodadadi A, Kazemeini M, Mortazavi Y. Enhanced $\mathrm{CO}$ sensitivity and selectivity of gold nanoparticles-doped $\mathrm{SnO}_{2}$ sensor in presence of propane and methane. Sens Actuators B 2008; 133: 352-6.

[8] Yang GW. Laser ablation in liquids: Applications in the synthesis of nanocrystals. Progr Mater Sci 2007; 52: 648-98.

[9] Tsuji T, Iryo K, Watanabe N, Tsuji M. Preparation of silver nanoparticles by laser ablation in solution: influence of laser wavelength on particle size. Appl Surf Sci 2002; 202: 80-5.

[10] Dolgaev SI, Simakin AV, Voronov VV, Shafeev GA, BozonVerduraz F. Nanoparticles produced by laser ablation of solids in liquid environment. Appl Surf Sci 2002; 186: 546-51.

[11] Tilaki RM, Iraji zad A, Mahdavi SM. Stability, size and optical properties of silver nanoparticles prepared by laser ablation in different carrier media. Appl Phys A 2006; 84: 215-9.

[12] Mafune F, Kohno J, Takeda Y, Kondow TJ. Nanoscale soldering of metal nanoparticles for construction of higher-order structures. Am Chem Soc 2003; 125: 1686-897.

[13] Liang C, Shimizu Y, Sasaki T, Koshizaki N. Synthesis of ultrafine $\mathrm{SnO}_{2-\mathrm{x}}$ nanocrystals by pulsed laser-induced reactive quenching in liquid medium. J Phys Chem B 2003; 107: 9220-25.

[14] Buffat P, Borel JP. Size effect on the melting temperature of gold particles. Phys Rev A 1976; 13: 2287-98.

[15] Takami A, Kurita H, Koda S. Laser-Induced size reduction of noble metal nanoparticles. J Phys Chem B 1999; 103: 1226-32.

[16] Briggs D, Seah MP. Practical surface analysis 1. Chichester: Wiley and Sons 1990.

[17] Tian C, Mao B, Wang E, et al. Simple strategy for preparation of core colloids modified with metal nanoparticles. J Phys Chem C 2007; 111:3651-7.

[18] Arabatzis IM, Stergiopoulos T, Andreeva D, Kitova S, Neophytides SG, Falaras P. Characterization and photocatalytic activity of $\mathrm{Au} / \mathrm{TiO} 2$ thin films for azo-dye degradation. J Catal 2003; 220: 127-35.

[19] Arias AC, Roman LS, Kugler T, Toniolo R, Meruvia MS, Hummelgen IA. The use of tin oxide thin films as a transparent electrode in PPV based light-emitting diodes. Thin Solid Films 2000; 371: 201-6.

[20] Ioannides T, Verykios XE. Charge transfer in metal catalysts supported on doped tio2: a theoretical approach based on metalsemiconductor contact theory. J Catal 1996; 161: 560-69.

[21] Izgaliev AT, Simakin AV, Shafeev GA, Bozon-Verduraz F. Intermediate phase upon alloying $\mathrm{Au}-\mathrm{Ag}$ nanoparticles under laser exposure of the mixture of individual colloids. Chem Phys Lett 2004; 390: 467-71. 